PROCEEDINGS OF THE

AMERICAN MATHEMATICAL SOCIETY

Volume 37, Number 1, January 1973

\title{
INVERTING SETS FOR FUNCTION ALGEBRAS
}

\author{
LARRY Q. EIFLER
}

\begin{abstract}
If $A$ is a function algebra on $X$, then we say that $X$ is an inverting set for $A$ if $f \in A$ and $f$ does not vanish on $X$ implies $f$ is invertible in $A$. We obtain results on inverting sets for tensor products and for extensions of $R(X)$ by real valued functions.
\end{abstract}

1. Introduction. Let $X$ be a compact Hausdorff space and let $C(X)$ denote the algebra of complex valued continuous functions on $X$ with supremum norm. Let $A$ be a function algebra on $X$. We say that $X$ is an inverting set for $A$ if $f \in A$ and $f$ does not vanish on $X$ implies $1 / f \in A$. We denote the Šilov boundary of $A$ by $\partial_{A}$ where $\partial_{A} \subseteq X$. We say that $X$ is the maximal ideal space of $A$ and write $M(A)=X$ if for each nonzero multiplicative linear functional $\phi$ on $A$ there is $x \in X$ satisfying $\phi(f)=f(x)$ for each $f \in A$.

Let $\Delta=\{z \in C:|z| \leqq 1\}$ and let $P(\Delta \times \Delta)$ denote the uniform closure in $C(\Delta \times \Delta)$ of the polynomials in $z$ and $w$. Then $\{(z, w) \in \Delta \times \Delta:|z|=1$ or $|w|=1\}$ and $\{(z, w):|z|=|w| \leqq 1\}$ are inverting sets for $P(\Delta \times \Delta)$. We generalize this result to tensor products of function algebras. Let $X$ be a compact subset of $C$ and let $R(X)$ denote the uniform closure in $C(X)$ of the rational functions with poles off $X$. If $\mathfrak{F}$ is a set of continuous real valued functions on $X$, then $X$ is an inverting set for the closed algebra generated by $R(X)$ and $\mathfrak{F}$. Finally, we give an example of a function algebra contained in the disc algebra for which $\Delta$ is not an inverting set.

2. Tensor products. Let $A$ and $B$ be function algebras on $X$ and $Y$ respectively. Let $A \otimes B$ denote the closed linear span in $C(X \times Y)$ of functions of the form $f \otimes g$ where $f \in A$ and $g \in B$ and $(f \otimes g)(x, y)=f(x) g(y)$. We next give two results which are generalizations to tensor products of the behavior of zero sets of functions in the bidisc algebra.

THEOREM 1. Suppose $A$ and $B$ are function algebras on $X=M(A)$ and $Y=M(B)$ respectively. Then $\left(\partial_{A} \times Y\right) \cup\left(X \times \partial_{B}\right)$ is an inverting set for $A \otimes B$.

Proof. Fix $F \in A \otimes B$ and assume $F$ does not vanish on $\partial_{A} \times Y$ or $X \times \partial_{B}$. Let $K=\{x \in X: F(x,-)$ is invertible in $B\}$. Notice that $K \supset \partial_{A}$ and

Received by the editors August 20, 1971.

AMS (MOS) subject classifications (1970). Primary $46 \mathrm{~J} 10$.

Key words and phrases. Function algebras, tensor products, inverting sets.

(c) American Mathematical Society 1973 
that $K$ is open in $X$. There exists $\varepsilon>0$ such that $|F(x, y)| \geqq \varepsilon$ for each $x \in X$ and $y \in \partial_{B}$. Suppose $x_{\alpha} \in K$ and $x_{\alpha} \rightarrow x_{0}$. Then $\left|F\left(x_{\alpha}, y\right)\right| \geqq \varepsilon$ for each $y \in Y$ so $\left|F\left(x_{0}, y\right)\right| \geqq \varepsilon$ for each $y \in Y$. Thus $K$ is closed and we apply the Šilov idempotent theorem to obtain $K=X$. Hence, $F$ is invertible in $A \otimes B$ since $M(A \otimes B)=X \times Y$.

THEOREM 2. Suppose $A$ and $B$ are function algebras on $X=M(A)$ and $Y=M(B)$ respectively. Suppose $f \in A$ and $g \in B$ satisfy $|f(x)|=|g(y)|=1$ for each $x \in \partial_{A}$ and $y \in \partial_{B}$ and $f(X)=g(Y)=\Delta$. Then $\{(x, y):|f(x)|=$ $|g(y)|\}$ is an inverting set for $A \otimes B$.

Proof. Suppose $F \in A \otimes B$ and $F$ does not vanish on $\{(x, y):|f(x)|=$ $|g(y)|\}$. We may assume that $A$ and $B$ are antisymmetric. Let $K=$ $\{x \in X: F(x, y) \neq 0$ if $|g(y)| \leqq|f(x)|\}$. It suffices to show $K=X$. By the Silov idempotent theorem, we only need to show that $K$ is open, closed and nonempty. Notice that $K$ is nonempty since there is $x \in X$ such that $f(x)=0$. Clearly, $X \backslash K$ is closed. Suppose $x_{\alpha} \in K$ and $x_{\alpha} \rightarrow x_{0}$. Set $r=$ $\left|f\left(x_{0}\right)\right|$. There is $\varepsilon>0$ such that $\left|F\left(x_{0}, y\right)\right|>\varepsilon$ for each $y$ satisfying $|g(y)|=r$. Hence, we may assume, by taking a subnet, that $\left|F\left(x_{\alpha}, y\right)\right| \geqq \varepsilon$ for each $y$ satisfying $|g(y)|=r$. Let $Z=\{y \in Y:|g(y)| \leqq r\}$. Then $B_{z}$, the closure of $B \mid Z$, has $Z$ as its maximal ideal space. By the local maximum modulus theorem, the Šilov boundary of $B_{Z}$ is contained in $\{y:|g(y)|=r\}$. Hence, $x_{\alpha} \in K$ gives $\left|F\left(x_{\alpha}, y\right)\right| \geqq \varepsilon$ for each $y \in Z$. Passing to the limit, we have $\left|F\left(x_{0}, y\right)\right| \geqq \varepsilon$ for each $y \in Z$ and so $x_{0} \in K$. Thus, $K$ is open, closed and nonempty.

Note. The Silov boundary of $P(\Delta \times \Delta)$ is not an inverting set but is the intersection of two inverting sets: $\{(z, w) \in \Delta \times \Delta:|z|=1$ or $|w|=1\}$ and $\{(z, w):|z|=|w| \leqq 1\}$.

3. Extensions of $R(X)$. If $X$ is a compact subset of $C$, then $P(X)$ denotes the uniform closure in $C(X)$ of the polynomials in $z, R(X)$ denotes the uniform closure in $C(X)$ of the rational functions with poles off $X$, and $A(X)$ denotes the algebra of continuous functions on $X$ which are analytic in the interior of $X$. If $A$ is a function algebra on $X$ and if $\tilde{F}$ is a set of continuous functions on $X$, then $A[\mathfrak{F}]$ denotes the closed algebra generated by $A$ and $\mathfrak{F}$. We prove the following theorem in this section.

THEOREM 3. Let $X$ be a compact subset of $C$. Suppose $A=R(X)$ or $A=$ $A(X)$ and let $\mathfrak{F}$ be a set of real valued continuous functions on $X$. Then $X$ is an inverting set for $A[\mathcal{F}]$.

We first prove the following lemma concerning the zeros of functions in $A(X)$. 
Lemma. Let $X$ be a compact subset of $C$. Suppose $f \in A(X)$ and $g \in C(X)$ such that $g$ does not vanish on $X$ and $|f-g|<|g|$ on $\partial X$, the boundary of $X$. Then $f$ does not vanish on $X$.

Proof. We may assume that $g$ is a polynomial in $x$ and $y$. Let $K=$ $\{x \in X:|f(x)-g(x)| \geqq|g(x)|\}$. Then $K$ is a compact subset of $X^{\circ}$, the interior of $X$. Let $G$ be a smoothly bounded open set such that $K \subseteq G$ and $\bar{G} \subseteq X^{\circ}$. Since $|f / g-1|<1$ on $\partial G$, there exists a smooth function $h$ on $\partial G$ such that $f=g e^{h}$ on $\partial G$. Set $n$ equal to the number of zeros of $f$ in $G$ and notice $d f \mid f=d g / g+d h$. Apply Green's theorem and the argument principle to obtain

$$
2 \pi i \cdot n=\int_{\partial G} \frac{d f}{f}=\int_{\partial G} \frac{d g}{g}+\int_{\partial G} d h=\iint_{G} d\left(\frac{d g}{g}\right)=0 .
$$

We conclude that $f$ does not vanish on $X$.

Proof of TheORem 3. Let $f \in A[\mathfrak{F}]$ and assume that $f$ does not vanish on $X$. Let $K$ be a maximal set of antisymmetry for $A$ [₹]. To show that $f$ is invertible in $A[\mathcal{F}$, we only need to show that $f \mid K$ is invertible in $A[\mathfrak{F}] \mid K$ by Bishop's antisymmetric decomposition theorem [1, p. 60]. Let $Y$ be the $A$ convex hull of $K$ in $X$. Let $B$ denote the closure of $A \mid Y$. Thus, $M(B)=Y$ and $B|K=A[\mathfrak{F}]| K$ since each member of $\mathfrak{F}$ is constant on $K$ and $K$ is a peak set for $A[\mathfrak{F}]$. Let $g$ be a member of $B$ satisfying $f=g$ on $K$. Since $f$ does not vanish on $Y$ and $K \supset \partial Y$, the Lemma implies that $g$ does not vanish on $Y$ and hence $g$ is invertible in $B$. We conclude that $f \mid K$ is invertible in $A[\mathfrak{F}]|K=B| K$.

4. Examples. In this section we give four examples concerning inverting sets. We begin by giving examples to show that Theorem 3 cannot be generalized to the case where $\tilde{F}$ is a single function of constant modulus or where the space $X$ is a polynomially convex subset of $C^{2}$. We give an example of a function algebra on a Cantor set $K$ such that $K$ is not an inverting set. We use this algebra to construct a function algebra $B$ contained in the disc algebra such that the closed unit disc is not an inverting set for $B$.

EXAmpLe 1. Let $A=P(\Delta)[f]$ where $f(z)=e^{i 2 \pi|z|}$. This algebra was considered by Wilken [3, Example 3.1]. The function $f$ is not invertible in $A$ and so $\Delta$ is not an inverting set for $A$. One can see this by noticing that

$$
\phi(g)=\int_{0}^{1} \frac{1}{2 \pi} \int_{-\pi}^{\pi} g\left(r e^{i \theta}\right) d \theta d r
$$

is a multiplicative linear functional on $A$ and $\phi(f)=0$. 
EXAmple 2. Let $X=\left\{\left(z, e^{i 2 \pi|z|}\right):|z| \leqq 1\right\}$ and let $\mathfrak{F}=\{f \in C(\Delta \times \Delta): f$ is real valued and $f=0$ on $X\}$. Then $\Delta \times \Delta$ is not an inverting set for $P(\Delta \times \Delta)[\widetilde{\Im}]$. Define $g$ on $\Delta \times \Delta$ by $g(z, w)=i 2 \pi|z|$. Then $e^{g}$ belongs to $A$ but, applying remarks in Example 1, one sees that $e^{g}$ is not invertible in $A$.

EXAMPLE 3. We construct a function algebra $A$ on a Cantor set $K$ such that $K$ is not an inverting set for $A$. Let $X$ denote the maximal ideal space of $L^{\infty}(T, m)$ where $T$ is the unit circle and $m$ is Lebesgue measure. We consider $H^{\infty}$, the algebra of bounded analytic functions on the open unit disc, as a subalgebra of $L^{\infty}$ and we denote the Gelfand map of $L^{\infty}$ onto $C(X)$ by $f \rightarrow \hat{f}$. If $g$ is a continuous function on $X$ and if $g>0$, then there is a bounded real valued Borel function $u$ on $T$ such that $\hat{u}=\log g$ and the function

$$
h(z)=\exp \left[\frac{1}{2 \pi} \int_{-\pi}^{\pi} \frac{e^{i \theta}+z}{e^{i \theta}-z} u\left(e^{i \theta}\right) d \theta\right]
$$

belongs to $H^{\infty}$ and satisfies the conditions $|h|=e^{u}$ and $|\hat{h}|=g$ (see [2, p. 182]).

Let $f_{0}=z$ and $d_{0}(x, y)=|\hat{z}(x)-\hat{z}(y)|$ for $x, y \in X$. Then $d_{0}$ is a pseudometric on $X$. Since $X$ is totally disconnected, there exist $f_{1}, f_{2}, \cdots$ in $H^{\infty}$ such that $\left|\hat{f}_{n}\right|$ has finite range and the pseudometric $d_{n}$ on $X$, defined by

$$
d_{n}(x, y)=\sum_{k=0}^{n} 2^{-k}\left|\hat{f}_{k}(x)-\hat{f}_{k}(y)\right|,
$$

has the property that each component of $\left(X, d_{n}\right)$ has $d_{n}$-diameter less than $1 / n$. Set $d(x, y)=\lim _{h \rightarrow \infty} d_{n}(x, y)$. Identifying points in $X$ which are $d$ distance zero apart, one obtains a compact metric space $Y$ such that $Y$ is totally disconnected. Let $A$ be the function algebra on $Y$ generated by the functions $\hat{f}_{0}, \hat{f}_{1}, \cdots$ which can be considered as defined on $Y$. Then $Y$ is not an inverting set since $f_{0}=z$ maps $Y$ onto $T$ and $z$ is not invertible in $H^{\infty}$. Now, set $K=\partial_{A}$. Finally, $K$ is a Cantor set since $K \subseteq Y$ and $K$ has no isolated points. If $y$ is an isolated point in $K$, then $\chi_{\{y\}}$ belongs to $A$ but $H^{\infty}$ has no idempotents except 0 and 1 .

EXAMPLE 4. Using Example 3, one may obtain a function algebra $B$ which is contained in the disc algebra such that $\Delta$ is not an inverting set for $B$. Let $A$ and $K$ be as in Example 3. Let $K^{\prime}$ be a Cantor set of measure zero in $T$ and let $\psi$ be a homeomorphism of $K$ onto $K^{\prime}$. Let

$$
B=\{f \in P(\Delta): f \circ \psi \in A\} .
$$

Then $B$ is a function algebra on $\Delta$ since $K^{\prime}$ is a peak interpolation set for $P(\Delta)$. Choose $f \in A$ such that $f$ is not invertible in $A$ but $f$ does not vanish on $K$. Since $K$ is a Cantor set, there is a continuous function $g$ on $K$ such that $e^{g}=f$. Choose $\bar{g} \in P(\Delta)$ such that $\bar{g} \circ \psi=g$. Then $\exp (\bar{g})$ belongs to $B$ 
and is not invertible in $B$ since $\exp (\bar{g} \circ \psi)=f$. Of course $\exp (\bar{g})$ does not vanish on $\Delta$.

\section{REFERENCES}

1. T. W. Gamelin, Uniform algebras, Prentice-Hall, Englewood Cliffs, N.J., 1969.

2. K. Hoffman, Banach spaces of analytic functions, Prentice-Hall Series in Modern Analysis, Prentice-Hall, Englewood Cliffs, N.J., 1962. MR 24 \#A2844.

3. D. R. Wilken, Maximal ideal spaces and A-convexity, Proc. Amer. Math. Soc. 17 (1966), 1357-1362. MR 34 \#3375.

Department of Mathematics, Louisiana State University, Baton Rouge, LOUISIANA 70803

Current address: Department of Mathematics, University of Missouri at Kansas City, Kansas City, Missouri 64110 